\title{
Electrostatically driven vacuum-encapsulated polysilicon resonators \\ Part I. Design and fabrication
}

\author{
Rob Legtenberg, Harrie A.C. Tilmans* \\ MESA Research Institute, University of Twente, PO Bax 217, NL-7500 AE Enschede, Netherlands
}

Received 1 July 1993; accepted in revised form 2 June 1994

\begin{abstract}
Basic design issues and a fabrication process based on surface-micromachining techniques for electrostatically driven vacuumencapsulated polysilicon resonators are presented. A novel freeze-drying method that does not require vacuum equipment is presented. Reactive sealing with LPCVD silicon nitride is used to create the evacuated cavity, resulting in cavity pressures close to the deposition pressure. Design issues regarding choice of materials, technology and layout are discussed. First experimental results, including an admittance plot of the one-port resonator and a plot indicating the dependence of the $Q$ factor on the resonator geometry and ambient pressure, are presented.
\end{abstract}

Keywords: Electrostatic excitation and detection; Surface-micromachining techniques; Polysilicon resonators; Vacuum encapsulation

\section{Introduction}

Resonant sensors are very attractive for the precise measurement of mechanical quantities such as pressure and force. They offer high sensitivity and resolution together with a semidigital output, i.e., a frequency [1-4]. The central part of the sensor is a vibrating mechanical element called the resonator, which provides the frequency (shift) output.

Surface-micromachined resonators that are operated in a flexural mode of vibration have been used by scveral rcsearch groups in the past [5-7]. Without taking special precautions, these resonators suffer from small signal responses as a result of a low mechanical quality factor. The low quality factor is a direct consequence of the introduction of a second surface in close proximity to the resonator surface. This leads to so-called squeezefilm damping, which can be observed for the normal vibrational motion of two closely spaced surfaces $[5,8]$. Squeeze-film damping becomes significant if the gap spacing is small compared to the lateral dimensions of the resonator. Evacuating the surroundings would elim-

*Present address: Katholieke Universiteit Leuven, Departement Elektrotechniek-ESAT, Kardinaal Mercierlaan 94, B-3001 Heverlee, Belgium. inate this effect. At the same time, unwanted environmental effects, such as changes in the density of the surrounding medium, humidity and dust, are eliminated as well. Thus, the resonator must be placed inside a vacuum housing. This was realized in a very elegant way by lkeda et al. in $1988[9,10]$. They fabricated single-crystalline silicon resonators housed locally in on-chip vacuum shells, fabricated using a self-aligned. selective epitaxial technology in combination with selective anisotropic etching and hydrogen evacuation techniques. The same concept, but based on a different fabrication technology, was presented by Guckel et al. in $1990[11,12]$. They employed the polysilicon/silicon dioxide sacrificial-layer etching technique to realize a similar structure.

This paper deals with electrostatically driven vacuumencapsulated polysilicon resonators. It forms Part I of a set of two papers. In Part I, design and processing issues are discussed, whereas Part II [13] deals with a discussion of the resonator performance, including a description of the theoretical model. The process is based upon the polysilicon/silicon dioxide sacrificiallayer etching technique. A novel freeze-drying procedure, which, as opposed to earlier reports, takes less time and does not require any vacuum equipment, is presented. For the excitation and detection of the 
vibration, the emphasis is on a single-element or oneport approach [14].

\section{Design issues}

\subsection{Basic stnucture}

The basic structure of the resonator is shown in Fig. 1. The resonator consists of a prismatic beam with a rectangular cross section and is housed in an evacuated cavity. From an electric viewpoint, the structure defines a four-terminal device. Both the one- $[6,14]$ and twoport approaches $[5,7,10]$ can be used for excitation and detection. Details of the electric driving scheme are presented in the accompanying paper [13].

Electrostatic excitation and detection has been employed [3-7]. This is attractive because of its simplicity and compatibility with micromachining and IC technologies. The simple structure makes the mechanical properties, and in particular the residual stress, much easier to control. Also, the scheme does not suffer from disturbing frequency shifts due to thermally induced axial loads. Moreover, no additional transducer materials are required as would be necessary for some other drive and detection schemes, such as piezoelectric transduction. Thus, the electrostatic scheme provides an excellent way to achieve an 'all-silicon device', which is attractive for minimizing differential thermal-expansion effects.

\subsection{The resonator}

The static and dynamic characteristics of the resonator are determined by its in-plane geometry and by its characteristic dimensions perpendicular to the plane $[4,13]$. Flexural beam resonators were designed with lengths of $210,310,410$ and $510 \mu \mathrm{m}$ and widths of 25 , 50 and $100 \mu \mathrm{m}$. The beam thickness is approximately $1.5 \mu \mathrm{m}$. Furthermore, a beam-to-substrate gap of approximately $1.2 \mu \mathrm{m}$ and a beam-to-cap gap of $1.5 \mu \mathrm{m}$ were chosen. These dimensions lead to theoretical values of the static capacitance (excluding any parasitics) in

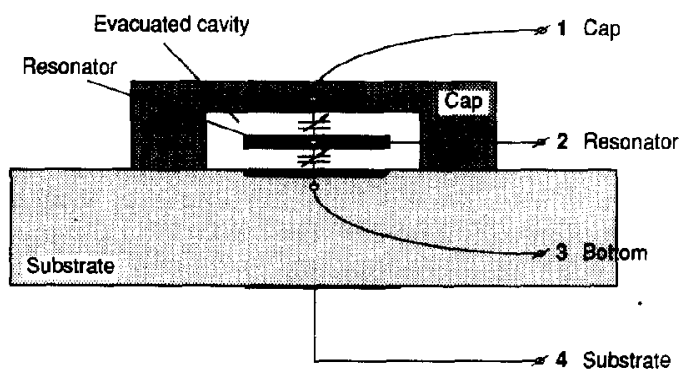

Fig. 1. Schematic cross section of a vacuum-encapsulated flexural beam resonator, depicted as a four-terminal device. the range $0.03-0.3 \mathrm{pF}$. Parasitic loads, which are mainly capacitivc, have to be kept very low or have to be compensated for in order to be able to detect the resonance [14]. The on-chip parasitic loads are mainly determined by the area of the bonding pads and the interconnecting wires. Thus, wire lengths were kept as short as possible and a relatively small bonding-pad area of $150 \mu \mathrm{m} \times 150 \mu \mathrm{m}$ was used. This resulted in measured parallel capacitances in the range $0.5-14 \mathrm{pF}$ (for the $210 \mu \mathrm{m}$ long beams), depending on the measurement configuration [13].

Chips measuring $6 \mathrm{~mm} \times 6 \mathrm{~mm}$ and including 12 different resonant structures were designed. As a minimum feature size, a rather conservative value of $5 \mu \mathrm{m}$ was used. Fig. 2 shows a typical layout of the resonator. A total of ten masks is needed for the fabrication. 'Drop-ins' of electric and mechanical diagnostics were used for characterization. They include checks for alignment accuracy, structures to measure the sheet and contact resistances and, further, ring- and buckledbeam structures to determine the residual strain [15].

\subsection{The sealing cap}

The mechanical stiffness of the sealing cap must be large enough to prevent excessively large deflections caused by the internal yacuum, which may lead to physical contact between the beam and the cap or the substrate. Thus, the upper limit for the cap width is determined by a given maximum deflection. The maximum deflection $w_{0}$ of a clamped rectangular plate with lateral dimensions $b \times a$ can be approximated as [16]

$w_{0} \approx \frac{0.00260 \Delta p a^{4}}{D}$ for $b>2 a$

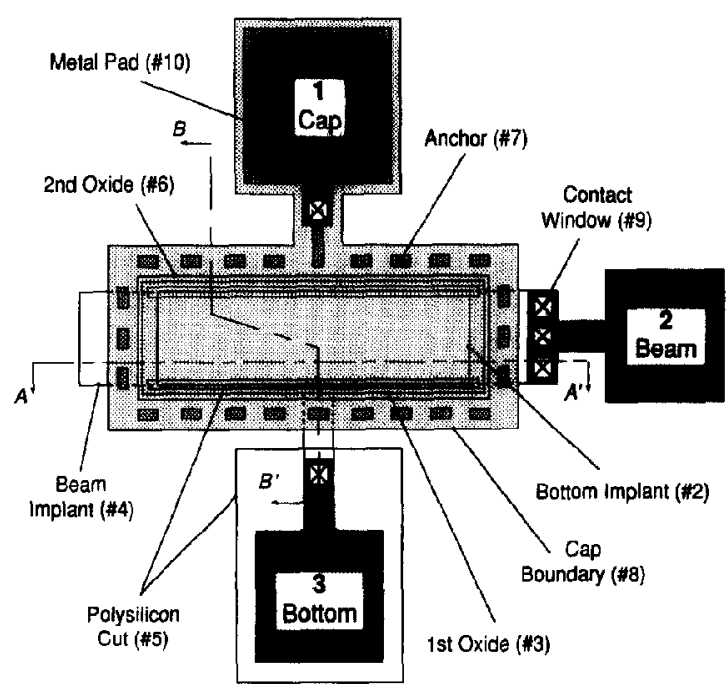

Fig. 2. Typical resonator layout. The resonator consists of a doubly supported beam. The in-plane beam dimensions are $410 \mu \times 100 \mu \mathrm{m}$. 
where $\Delta p$ is the difference in pressure of the cavity interior and the ambient and $D$ is the flexural rigidity of the plate. The coefficient 0.00260 depends on the length over width ratio $b / a$ and is within two per cent accuracy if this ratio is larger than two. For a cap thickness of $2.5 \mu \mathrm{m}$, this leads to an upper limit of the width of approximately $150 \mu \mathrm{m}$ if the maximum cap deflection should not exceed $0.5 \mu \mathrm{m}$. Finite-element analyses of a more realistic cap structure have indicated an increase of the maximum deflection of $20-30 \%$ due to small corrugations that are a result of the fabrication process (e.g., see Fig. 5(h)). This leads to a smaller value for the upper limit of the width. The cap thickness used is approximately $2.6 \mu \mathrm{m}$, which is sufficient to keep the inward deflection after sealing below $0.5 \mu \mathrm{m}$ for $100 \mu \mathrm{m}$ wide beams.

\section{Technology and materials}

\subsection{Surface micromachining}

Surface micromachining is used to fabricate the polysilicon resonant structures. The technology relies on the construction of mechanical parts from a thin-film material in combination with a sacrificial-layer thin film that is used as a spacer between the structural parts $[5,17,18]$. After selective chemical removal of the sacrificial layer, freely movable members remain. Surface micromachining offers small dimensions and a good dimensional control, especially in the thickness direction. Further, a very elegant way to fabricate evacuated cavities by means of surface-micromachining techniques has recently been presented $[17,19]$.

\subsection{Materials}

Taking planar silicon thin-film technology as a starting point, the aim is to develop an 'all-silicon device'. This provides a good thermal match with the silicon substrate and, further, allows IC-compatible processing. Fabrication is based on the polysilicon/silicon dioxide sacrificial-layer technique.

To obtain a high sensitivity of the frequency per unit applied strain, i.e., the gauge factor, slender beams are required with a low residual strain [4]. A tensile strain is desired to minimize the possibility of buckling. A tensile strain in the range $10-100 \mathrm{ppm}$ is considered to be optimal. The residual stress of polysilicon can be well controlled provided that proper deposition conditions and annealing cycles are used [15,19-21]. Polysilicon with a low tensile strain can be obtained by low-pressure chemical vapour deposition (LPCVD) from silane $\left(\mathrm{SiH}_{4}\right)$ at processing temperatures close to the temperature of transition from amorphous to (poly)crystalline layers $\left(\approx 580{ }^{\circ} \mathrm{C}[21]\right)$. Thermal an- nealing in nitrogen converts the as-deposited built-in compressive strain into a controllable tensile strain depending on the annealing temperature [15].

The electrodes of the driving capacitor are defined by locally doping the silicon substrate, the polysilicon beam and the cap. In order to prevent doping of unwanted places, undoped oxides must be used as the sacrificial-layer material. Undoped low-frequency (50 $\mathrm{kHz}$ ) plasma-enhanced chemical vapour deposition (PECVD) oxides provide an acceptable compromise between a good step coverage and a high sacrificiallayer etch rate. Doping is done by ion implantation of boron. Boron results in lower sheet resistivities in polysilicon than phosphorus and shows less segregation at the grain boundaries [22]. Boron implantation may, however, affect the mechanical properties of the polysilicon layer. Electrical insulation of the cap, the resonator and the substrate is achieved by using a separating layer of LPCVD silicon nitride. The layer also serves as the anchor material for the different polysilicon layers, as indicated in Fig. 3(a), (b). The capacitance and the conductivity between the different terminals provide a first indication of the integrity of the insulating layers and whether the beams are free standing or not.

\subsection{Sticking and freeze drying}

After etching the sacrificial layers in an undiluted (49\%) HF solution, a specific drying procedure is required to prevent sticking of the free structural members to the substrate. Standard drying procedures such as dry spinning or air drying produce surface-tension forces that cause 'pull-down' of the thin-film structures. Once contact has been made, other forces like van der Waals
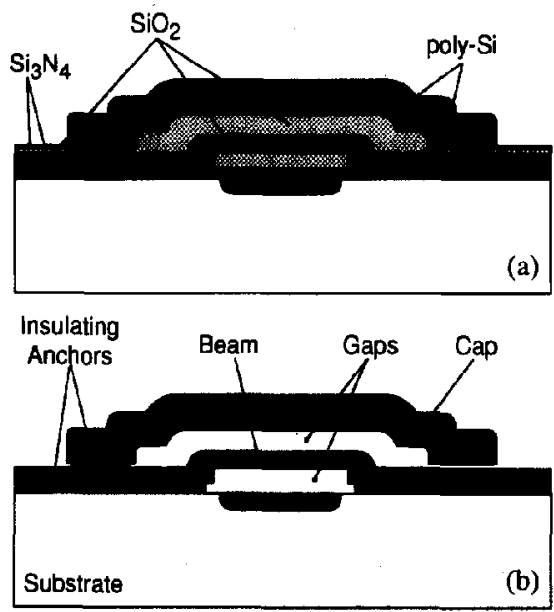

Fig. 3. Schematic of the electrical insulation between the resonant beam, the substrate and the cap using silicon nitride anchors; (a) prior to and (b) following the sacrificial layer etch. 
forces, electrostatic forces, hydrogen bridging and chemical reactions come into play, resulting in permanent attachment of the structures to the substrate [23-25]. An elegant way to solve this so-called sticking problem is 'freeze drying'. The freeze-drying method was developed as a drying method for biological specimens by Boyde and Wood in 1969 [26]. Guckel et al. [24] and later Takeshima et al. [27] implemented this technique for sacrificial-layer etching of surface-micromachined structures. They describe a process whereby a final rinsing agent is frozen and subsequently sublimated in a few hours under vacuum conditions.

In this paper a new and faster freeze-drying technique is presented. It does not require any vacuum equipment but instead can be performed under atmospheric conditions. Cyclohexane, which freezes at about $7{ }^{\circ} \mathrm{C}$, is used as the final rinsing agent. Freezing and subsequent sublimation are readily accomplished by placing the substrate under a nitrogen flow on a regulated Peltier element with a temperature below the freezing point (see Fig. 4). The total time for the freeze-sublimation process depends on the geometry of the sample and is typically $\mathbf{5 - 1 5} \mathrm{min}$ for the structures described in this paper.

Details of the rinse-freeze-sublimation procedure are as follows: after sacrificial-layer etching in an undiluted (49\%) HF solution, a dilution rinse in DI water is performed to remove the etchant. Isopropyl alcohol (IPA) is added to keep the hydrophobic wafer surface wet after removal from the solution. The wafers are placed in another beaker containing IPA and next are placed in cyclohexane, the final rinsing agent. The IPA serves as an intermediate mixing agent. After rinsing the wafer in cyclohexane, it is placed upon a Peltier element that has already been cooled to $5^{\circ} \mathrm{C}$. A nitrogen flow aids the sublimation process by removing cyclohexane vapours and preventing condensation of water. After sublimation is complete, the Peltier element is raised to room temperature, which completes the fabrication of the free-standing structures. No residues have been observed. Microbridges with aspect ratios

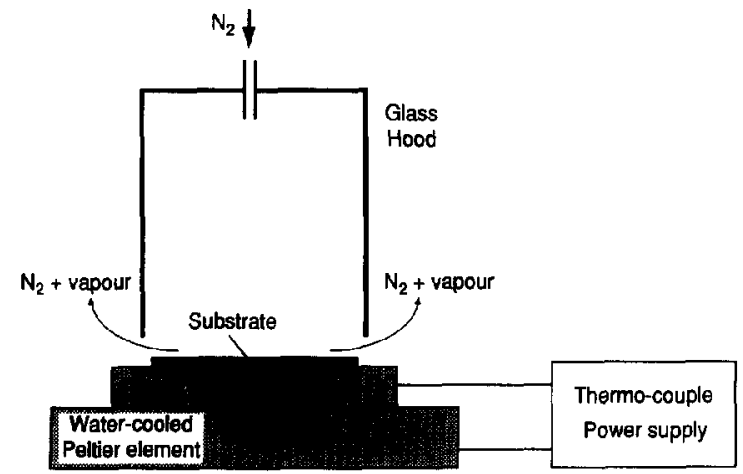

Fig. 4. Schematic of the experimental freeze-drying set-up. (length/thickness) as high as 2000 , at a gap spacing of $1 \mu \mathrm{m}$, were obtained by this freeze-drying procedure.

\subsection{Reactive sealing}

The reactive sealing technique as developed by Burns and Guckel $[17,19]$ is an elegant way to create vacuumsealed cavities. It exploits the difference in geometry between the small etch channels and the larger cavities to close off the latter by growing a thin film in the channels. Good step coverage is favourable for good sealing properties, but also results in the deposition of a thin layer inside the cavity before the etching channels are closed. In the case of the electrostatic driving scheme, a material with good insulating properties must be chosen, otherwise the electric terminals labelled 1,2 and 3 in Fig. 1 will be short circuited. This would prohibit the build-up of an electric field, thus making electrostatic excitation and detection impossible. Furthermore, it is preferable to use a material with a tensile residual strain in order to minimize the possibility of the resonator buckling. LPCVD of silicon nitride was chosen as the reactive sealing process.

The residual pressure inside the cavity after sealing is of importance with respect to the attainable quality factors $[3,4,28]$. The cavity pressure after sealing is a function of the processing pressure and temperature, the gas flows, the reaction products and subsequent (out)diffusions. The overall chemical reaction for the deposition of silicon nitride from dichlorosilane (DCS) and a surplus of ammonia $\left(\mathrm{NH}_{3}\right)$ is [29]:

$$
\begin{aligned}
& 3 \mathrm{SiCl}_{2} \mathrm{H}_{2}+(4+x) \mathrm{NH}_{3} \longrightarrow \\
& \mathrm{Si}_{3} \mathrm{~N}_{4} \downarrow+6 \mathrm{HCl} \uparrow+6 \mathrm{H}_{2} \uparrow+x \mathrm{NH}_{3} \uparrow
\end{aligned}
$$

This reaction clearly indicates that the number of gaseous molecules increases. This means that the cavity pressure will be higher than the deposition pressure, whence the etch channels are closed off. According to Boyle Gay-Lussac's law for an ideal gas, the final cavity pressure $p_{\text {cavity }}$ can be expressed as

$p_{\text {cavity }}=p_{\text {process }} \frac{V_{\text {unsealed }}}{V_{\text {scalcd }}} \frac{T_{0}}{T_{\text {process }}} \frac{n_{\text {right }}}{n_{\text {left }}}$

where $T_{0}$ and $T_{\text {process }}$ denote room temperature and the processing temperature, respectively, $V_{\text {unsealed }} / V_{\text {sealed }}$ denotes the ratio of the volume of the cavity prior to and after sealing, $p_{\text {process }}$ denotes the processing pressure and $n_{\text {right }} / n_{\text {left }}$ denotes the mole ratio of gaseous products and reactants.

To get an idea of the final cavity pressures, a DCS: $\mathrm{NH}_{3}$ flow ratio of 3:4 (i.e., $x=0$ ) and no subsequent reactions and diffusions after sealing are assumed. For the reaction given by Eq. (2) the mole ratio equals $12 / 7$. The ratio $V_{\text {unsealed }} / V_{\text {sealed }}$ results in a slight increase of the sealing pressure and is due to a volume reduction as a result 
of the inward deflection of the sealing cap. This deflection is generally undesirable and is therefore kept small, resulting in a volume ratio close to unity. For a processing pressure of $200 \mathrm{~m}$ Torr $(0.26 \mathrm{mbar})$ and a deposition temperature of $800^{\circ} \mathrm{C}$, Eq. (3) predicts a sealing pressure of about 100 mTorr ( 0.13 mbar). A more dedicated process performed at a pressure of 20 mTorr, a temperature of $850^{\circ} \mathrm{C}$ and the same gas-flow ratio as indicated above leads to a sealing pressure of approximately $10 \mathrm{mTorr}$, but exhibits a lower deposition rate. By using a flow ratio DCS: $\mathrm{NH}_{3}=1: 3$, a surplus of ammonia results (i.e., $x=5$ ) and the mole ratio is equal to $17 / 12$. This leads to a lowering of the final cavity pressure from 100 to 83 mTorr. Extending the surplus to infinity results in a final cavity pressure of $58 \mathrm{~m}$ Torr. These simple examples indicate that the mole ratio is not the determining factor, but, instead, the processing pressure and the processing temperature are the most important parameters in determining the final cavity pressure. Low final cavity pressures using a LPCVD silicon nitride sealing layer are obtained at a high deposition temperature and a low deposition pressure.

\section{Fabrication sequence}

A scheme of the fabrication process is shown in Fig. 5. The substrates are (100) n-type (phosphorus doped, $5-10 \Omega \mathrm{cm}$ ) silicon wafers. The fabrication sequence begins with a dry etching step to define the alignment marks into the silicon substrate (Mask \#1). Next, boron is implanted (dose $=5 \times 10^{15} \mathrm{~cm}^{-2}$, energy $=100 \mathrm{keV}$ ) through a photoresist mask (Mask \#2) to define the bottom electrode (see Fig. 5(a)). This is followed by a blanket phosphorus implant (dose $=1 \times 10^{15} \mathrm{~cm}^{-2}$, energy $=150 \mathrm{keV}$ ) of the backside of the wafer to obtain a good backside contact. After plasma ashing the photoresist, the wafer is cleaned and is given an HF dip ( 1 min in $\mathrm{HF}: \mathrm{H}_{2} \mathrm{O}=1: 100$ ) to remove the native oxide. A $200 \mathrm{~nm}$ thick, stoichiometric LPCVD silicon nitride layer is grown for the insulating (anchoring) layers (see also Fig. 3(a), (b)).

The first sacrificial layer consists of a $1.18 \mu \mathrm{m}$ thick PECVD oxide layer, grown from a $2 \% \mathrm{SiH}_{4}$ (in $\mathrm{N}_{2}$ ) and $\mathrm{N}_{2} \mathrm{O}$ mixture. This layer is densified for $30 \mathrm{~min}$ at $800{ }^{\circ} \mathrm{C}$ in a nitrogen ambient to improve the thermal stability. Patterning of the oxide layer (Mask \#3) is done by wet chemical etching using buffered HF (BHF). After cleaning, the wafers are given an HF dip to remove the native oxide and to ensure proper attachment of the next LPCVD polysilicon layer. This polysilicon layer is $1.50 \mu \mathrm{m}$ thick and is grown by pyrolysing silane $(50 \mathrm{sccm})$ at a temperature of $590^{\circ} \mathrm{C}$ and a pressure of $250 \mathrm{~m}$ Torr, giving a deposition rate of approximately $6.3 \mathrm{~nm} / \mathrm{min}$ (see Fig. 5(b)).
The following step is the implantation of boron (dose $=1 \times 10^{16} \mathrm{~cm}^{-2}$, energy $=150 \mathrm{keV}$ ) through a photoresist mask (Mask \#4) to define the electrode of the resonant beam. After plasma ashing the photoresist and subsequent cleaning, the length and width of the beams are defined by reactive ion etching (RIE) in an $\mathrm{SF}_{6}$ plasma (Mask \#5). At the same time, the polysilicon is removed at the places for the substrate metallization contacts (see Fig. 5(c)).

This is followed by the deposition, densification and patterning (Mask \#6) of the second PECVD sacrificial oxide layer (see Fig. 5(d)). The thickness of the oxide layer is approximately $1.55 \mu \mathrm{m}$.

Wet oxidation at $1000^{\circ} \mathrm{C}$ for $8 \mathrm{~min}$ forms a $80 \mathrm{~nm}$ thick oxide layer in which the anchors (and etch channels) are defined using BHF (Mask \#7). For electric insulation, a $50 \mathrm{~nm}$ LPCVD silicon nitride layer is grown, which serves as the insulation layer between the beam and cap electrodes (see Fig. 5(e) and Fig. 3(a), (b)).

The next step involves cleaning and removal of the native oxide, followed by LPCVD of a polysilicon layer for the cap with a thickness of approximately $2.64 \mu \mathrm{m}$. A blanket boron implantation (dose $=1 \times 10^{16} \mathrm{~cm}^{-2}$, energy $=100 \mathrm{keV}$ ) forms the conductor for the cap electrode. Patterning of the doped polysilicon layer defines the cap structure (Mask \#8, see Fig. 5(f)). This is done using $\mathrm{RIE}$ in an $\mathrm{SF}_{6} / \mathrm{N}_{2}$ plasma, which gives an etch selectivity of 40:1 for polysilicon over silicon oxide. The etching is stopped as soon as the underlying silicon nitride and silicon oxide double layer of the etch channels is exposed. From this point on, thick photoresist layers $(3 \mu \mathrm{m})$ have to be used to ensure sufficient step coverage. Annealing in a nitrogen ambient for $1 \mathrm{~h}$ at a temperature of $1000{ }^{\circ} \mathrm{C}$ is necessary for the electric activation of the implanted boron and, moreover, to obtain a low tensile strain in the polysilicon layers.

Following a thorough cleaning step, the sacrificiallayer etch and subsequent freeze-drying procedure are performed. Sacrificial-layer etching is done in an undiluted (49\%) HF solution and takes about two hours. Quenching in DI water is done for $30 \mathrm{~min}$ and next IPA is added. The wafers are rinsed for $1 \mathrm{~h}$ in an IPA solution, followed by a final rinse cycle in cyclohexane for $1 \mathrm{~h}$. Next, the freeze drying is carried out as described in the previous section (see Fig. 5(g) and Fig. 4). The mechanical test structures can now be examined to check whether the mechanical stress is in the region of interest and whether sacrificial layer etching and freeze drying have been successful. LPCVD of $190 \mathrm{~nm}$ stoichiometric silicon nitride is used to close off the etch channels. The surface passivation of the pn-junction of the bottom electrodes in the substrate is important to keep the leakage currents of the junction acceptably small. Dry thermal oxidation is the best way to ac- 

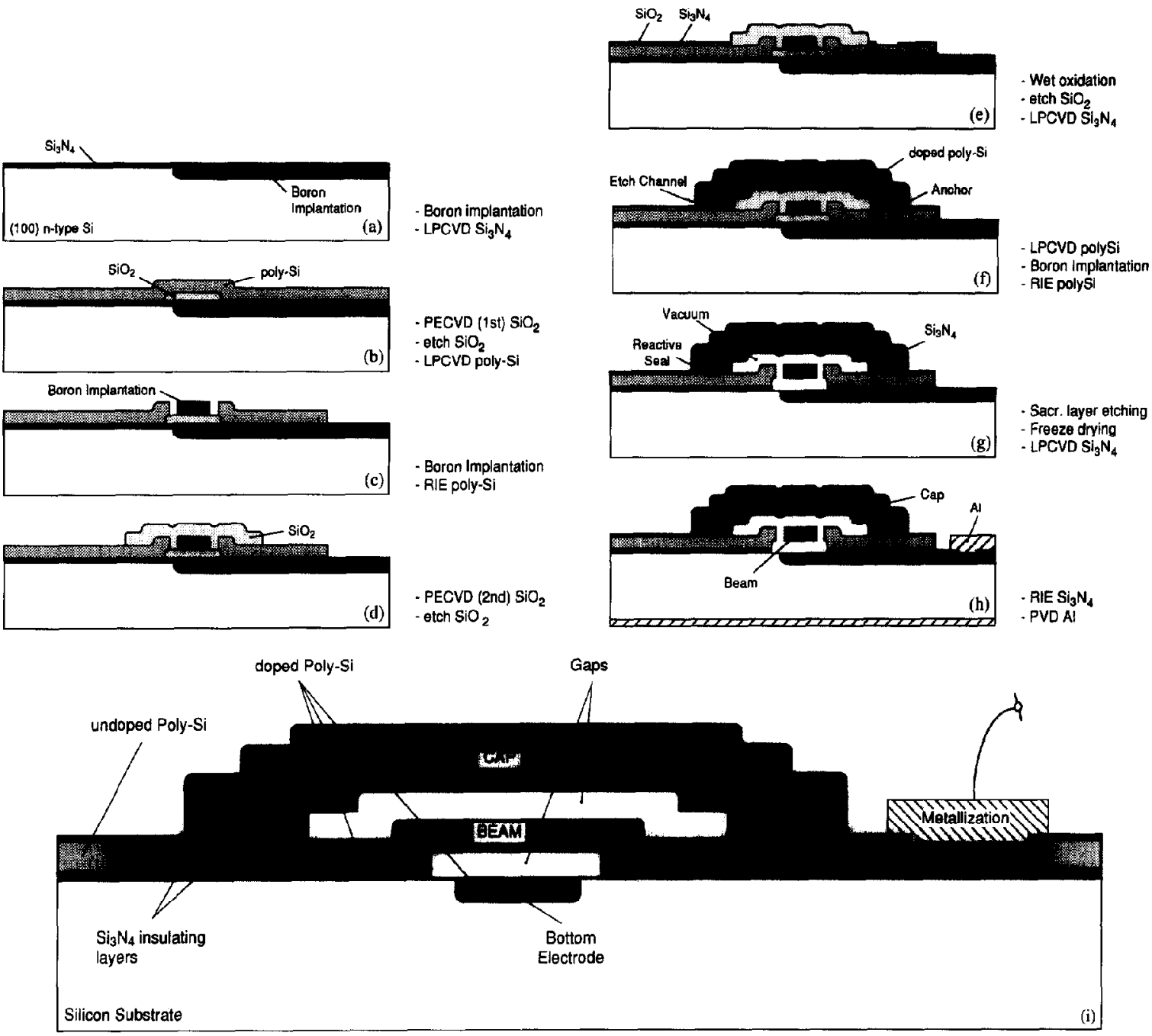

Fig. 5. Processing sequence for sealed polysilicon resonaturs. The different stages of the process are explained in the lext: (a)-(h) crosssectional view B-B'; (i) cross-sectional view A-A of Fig. 2.

complish this, but experiments revealed buckled beams even after growing oxide layers as thin as $10 \mathrm{~nm}$ at a temperature of $800^{\circ} \mathrm{C}$. It was found that the leakage currents of the pn-junctions that are passivated with silicon nitride are of the order of $10 \mu \mathrm{A} \mathrm{cm}{ }^{-2}$, which is considered to be satisfactory.

The contact windows for the substrate, beam and cap electrodes are defined using $\mathrm{RIE}$ in a $\mathrm{CHF}_{3} / \mathrm{O}_{2}$ plasma (Mask \#9). Metallization consists of a $1 \mu \mathrm{m}$ thick aluminium layer. Prior to the metallization, the thin films that have accumulated on the backside of the wafer are stripped by means of RIE in $\mathrm{SF}_{6}$ and $\mathrm{CHF}_{3}$ plasmas. Metal patterning (Mask \#10) is done by wet chemical etching. The final step is a $5 \mathrm{~min}$ anneal in an $\mathrm{N}_{2} / \mathrm{H}_{2} \mathrm{O}$ ambient at $450{ }^{\circ} \mathrm{C}$ to obtain good ohmic contacts. Final cross sections of the sealed resonator are shown in Fig. 5(h) and (i).

\section{First results}

The SEM photograph of Fig. 6(a) shows a top view of a sealed resonator. To inspect the inside of the cavity for residues, and check whether or not the beams are free standing, caps have been manually removed. This was done by peeling off a carefully attached tape. The cavity inside turned out to be clean and displayed free-standing beams having smooth surfaces (see Fig. 6(b)). Cross-sectional views of the resonator are shown in Fig. 6(c), (d). These SEM photographs clearly indicate (part of) the free-standing beam and the shape of the cap (compare Fig. 5(h)).

\subsection{Electrical and electromechanical behaviour}

Electrical measurements indicated sheet resistances of 24,30 and $22 \Omega /$ square for the bottom electrode, 

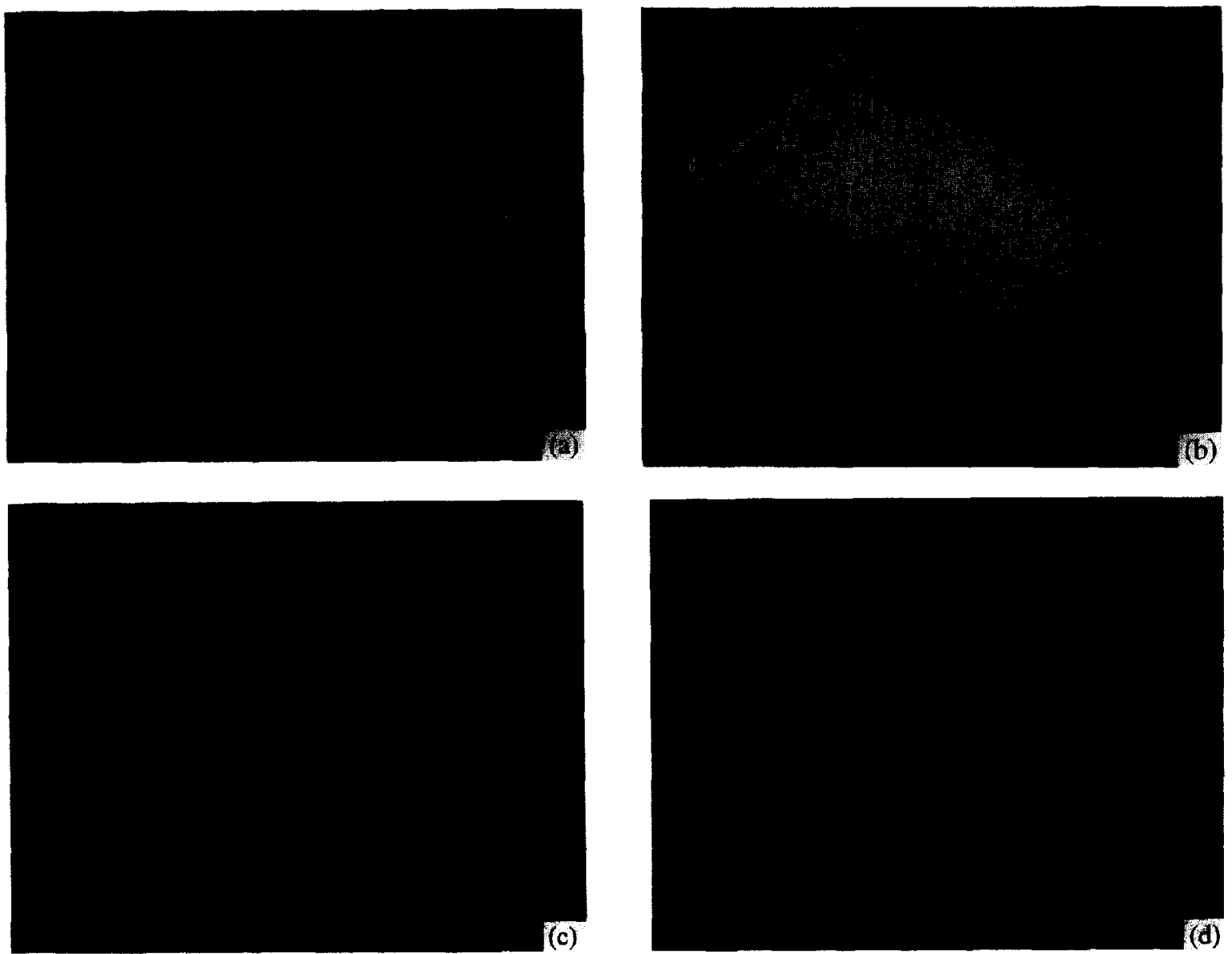

Fig. 6. SEM photographs of sealed polysilicon resonators; (a) top view of a resonator; (b) resonator with cap removed; (c) cross-sectional view; (d) magnified view of part of the cross section.

the resonator electrode and the cap electrode, respectively. The contact resistance turned out to be about $1 \Omega$ for a $20 \mu \mathrm{m} \times 20 \mu \mathrm{m}$ contact for all three electrodes. Standard $I-V$ measurements used to check the integrity of the nitride insulating anchors revealed high obmic (GRs) anchors. The residual strain has been extracted from the polysilicon ring and buckledbeam diagnostics and is within the range $-8 \times 10^{-7}$ (compressive) to $+6 \times 10^{-5}$ (tensile).

First tests aimed at finding the resonance frequency have indicated that care must be taken with respect to probing the samples. Static charges induce electric forces that cause 'pull-in' of the beams, resulting in permanent attachment of the latter to the cap or to the substrate. This phenomenon was observed while testing uncapped resonators. Fig. 7 shows a typical example of a measured Bode plot showing the conductance and the susceptance as functions of the driving frequency of a $210 \mu \mathrm{m}$ long polysilicon beam resonator. Details of the measurement set-up are described in the accompanying paper [13].

\subsection{Quality factor and sealing pressure}

Vacuum sealing provides pressures in the molecular regime, where the gas molecules are not interacting with each other. Energy losses due to viscous damping are negligible in this pressure regime. The damping mechanisms that remain include losses due to individual collisions of the gas molecules with the resonating surfaces (momentum damping), losses into the mount and intrinsic (material) damping. If momentum damping is considered to be the dominant loss mechanism and if the resonator was operating in free space, the mechanical quality factor as a function of the ambient pressure $p$ can be expressed as [28]

$Q_{n}=\frac{3}{8 \sqrt{6}} \frac{\alpha_{n}^{2}}{p}\left[\frac{h}{l}\right]^{2}\left(\frac{\pi E \rho R T}{M}\right)^{1 / 2}$

where $\alpha_{n}$ is a dimensionless constant depending on the edge conditions and on the mode of vibration $n\left(\alpha_{1}=4.73\right.$ for a clamped-clamped beam [4]), $h$ and $l$ are the 


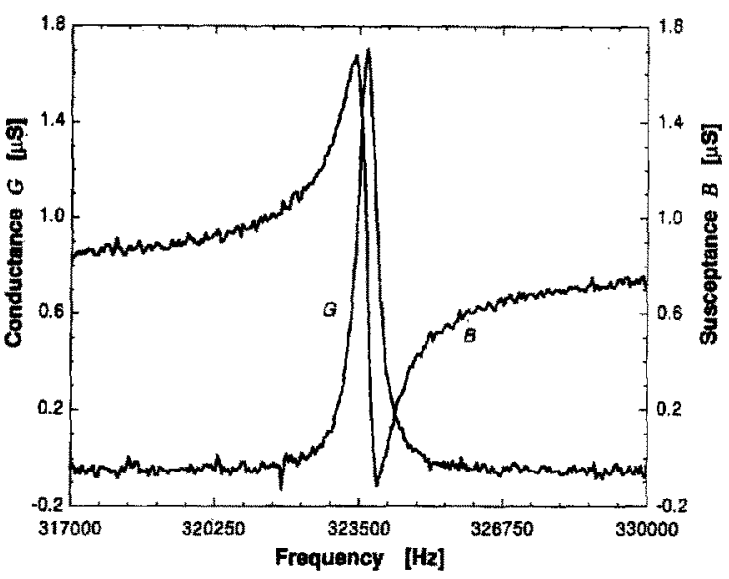

Fig. 7. Admittance Bode plot of an electrostatically driven vacuumencapsulated polysilicon resonator in a one-port configuration [13]. Resonator dimensions are $210 \mu \mathrm{m} \times 100 \mu \mathrm{m} \times 1.5 \mu \mathrm{m}$. The observed resonance frequency is $323682 \mathrm{~Hz}$.

thickness and the length of the resonator, respectively, $E$ and $\rho$ are the Young's modulus and the specific mass of the resonator material, respectively, $R\left(\approx 8.31 \mathrm{~J} \mathrm{~K}^{-1}\right.$ $\left.\mathrm{mol}^{-1}\right)$ is the universal gas constant, $T$ is the absolute temperature and $M$ is the molecular mass of the gas. Typical values for the $Q$-factor, assuming a nitrogen atmosphere, of a polysilicon resonator with an aspect ratio $l / h=300$ as obtained from Eq. (4) are $3900 / p$, where $p$ is the pressure in mbar.

The mechanical quality factor of the one-port resonators can be extracted from the measured Bode plot (as explained in Ref. [13]) and turned out to be a function of the resonator length. Typical values of the quality factor are $600,150,45,30$ for the 210,310 , 410 and $510 \mu \mathrm{m}$ long beams, respectively. The quality factor varied across the wafer, which is most likely due to variations of the sealing pressure and not to variations of the film properties and critical dimensions (e.g., the thickness). The latter is concluded, since the spread in the resonance frequencies across the wafer was within a few percent [13].

In order to measure the quality factor as a function of pressure, a leak must be introduced to the cavity. Fracturing the cap is not considered to be the best solution, since this would remove the upper gap and thus would give misleading results if effects such as squeeze-film damping are significant. Therefore, a specially designed 'dual cavity structure' has been used to measure the quality factor as a function of the pressure inside the cavity. The structure consists of two cavities that are interconnected through a narrow channel. One cavity houses the resonator. By fracturing the other cavity and placing the structure in a vacuum chamber, the pressure of the cavity containing the resonator could be regulated by the external vacuum system. In this way, the resonator is still sandwiched between two air gaps, and thus forms a realistic representation of the actual situation. The results are shown in Fig. 8. The graph indicates that the quality factor is inversely proportional to the pressure (as predicted by Eq. (4)) in the range $0.01-1.0$ mbar. This suggests that in this pressure regime, momentum damping is the dominating loss mechanism. However, measured $Q$-values are more than one order of magnitude smaller than predicted and some form of 'enhanced momentum damping' exists. This discrepancy has also been observed by Zook et al. [30]. The primary cause for the discrepancy is the fact that the assumption of free space surrounding the resonator, which is used to derive Eq. (4), is not true. Squeeze-film effects have to be taken into account, even for pressures in the molecular regime $[5,8]$. This is further strengthened by the distinct values for $Q$ that are measured for a $310 \mu \mathrm{m}$ long beam with (i.e., dual structure) and without a cap, or in other words, with two gaps and a single gap, respectively. The $Q$ factor for the structures with two gaps was approximately a factor of two to three smaller than the $Q$-factor of the structures with a single gap. Details of the theory of squeeze-film effects are beyond the scope of this paper and reference is made to the literature, e.g., Ref. [8] and references therein. The stationary value of the $Q$-factor at very low pressures is determined by intrinsic damping effects and by losses into the mount. Measurements have indicated stationary $Q$-factors close to 18000 (see Fig. 8).

The dual cavity structure described above is also used to determine the final cavity pressure. By matching the Bode plots of a sealed resonator and the dual cavity resonator, a first indication of the cavity pressure could

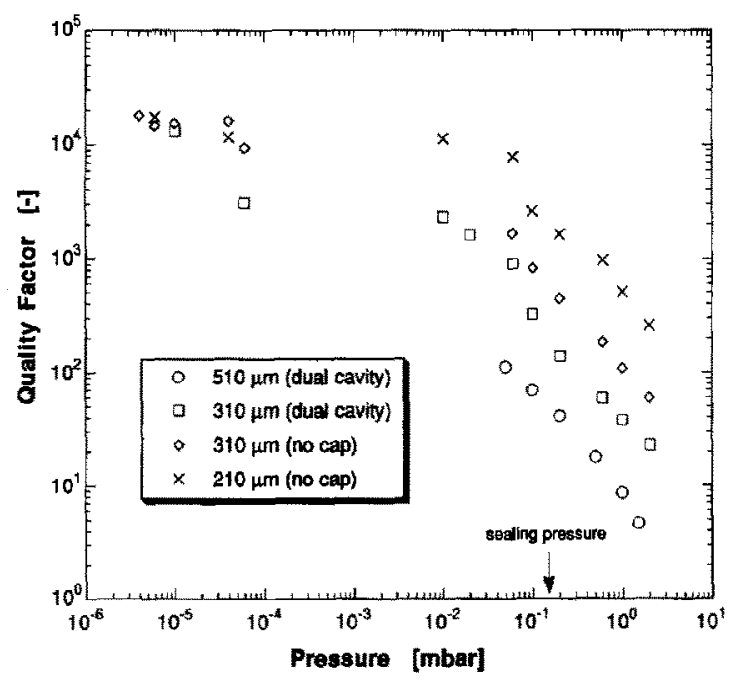

Fig. 8. Measured quality factor as a function of ambient pressure for beams with and without cap and for different beam lengths. Beam thickness and width are 1.5 and $100 \mu \mathrm{m}$, respectively; lower and upper gap spacings are approximately 1.2 and $1.5 \mu \mathrm{m}$, respectively. 
be obtained. This revealed sealing pressures in the range $0.1-0.2$ mbar, which is in agreement with the predicted value ( $\approx 0.13$ mbar) from Eq. (3).

More experimental data together with a theoretical model of the resonator behaviour are given in the accompanying paper [13].

\section{Conclusions}

Resonators housed in evacuated cavities have been successfully fabricated with surface micromachining using sacrificial-layer spacers. Low-strain fine-grained LPCVD polysilicon has been used for the structural parts and undoped PECVD silicon oxide as the sacrificial-layer material. Electrostatic excitation and detection was used as the driving and sensing mechanism. Electrical insulation of the different electrodes is accomplished by means of LPCVD silicon nitride layers. A novel freeze-drying method using cyclohexane has been developed to prevent sticking. The advantages of this procedure as compared to other known freezedrying procedures are its enhanced speed and the fact that no vacuum equipment is required. Reactive sealing with LPCVD silicon nitride yields cavity pressures close to the processing pressure. First measurements have indicated the typical resonance characteristics that are expected for resonators operated in a one-port configuration. Quality factors are determined by enhanced momentum damping (for pressures in the molecular regime) due to squeeze-film effects, and turn out to be inversely proportional to the ambient pressure for moderate sealing pressures (0.01-1.0 mbar). Measured sealing pressures are estimated to be in the range $0.1-0.2$ mbar, resulting in quality factors close to 600 for the $210 \mu \mathrm{m}$ long beams. A more dedicated sealing procedure at lower deposition pressures and/or higher temperatures is required to obtain cavity pressures in the regime where the quality factor is independent of pressure and is solely determined by intrinsic losses and losses into the mount. Subsequent annealing at elevated temperatures may further reduce the sealing pressure due to outdiffusion of gaseous species. Measurements have indicated that quality factors close to 18000 are feasible at pressures below 0.01 mbar.

\section{Acknowledgements}

The authors acknowledge Dr Miko Elwenspoek, Professor Dr Jan Fluitman and Professor Dr Theo Popma of the Micromechanics Group for their interest and support. Further, they thank the staff of the MESA Laboratory of the University of Twente, in particular Arie Kooy for mask fabrication, and Eddy de Ruiter, Tom Aarnink and Gerrit Boom for doing the ion implantations. Also they would like to thank Dick Ekkelkamp for his technical support and Hans Schurer for doing the $Q$-factor measurements. This research is part of the programme of the Dutch Foundation for Fundamental Research on Matter (FOM) and is sponsored by the Dutch Technology Foundation (STW).

\section{References}

[1] R.M. Langdon, Resonator sensors - a review, J. Phys. E: Sci. Instrum., 18 (1985) 103-115.

[2] R.T. Howe, Resonant microsensors, Proc. 4th Int. Conf. SolidState Sensors and Actuators (Transducers '87), Tokyo, Japan, June 2-5, 1987, pp. 843-848.

[3] G. Stemme, Resonant silicon sensors, J. Micromech. Microeng., 1 (1991) 113-125.

[4] H.A.C. Tilmans, M. Elwenspoek and J.H.J. Fluitman, Micro resonant force gauges, Sensors and Actuators A, 30 (1992) 35-53.

[5] R.T. Howe and R.S. Muller, Resonant-microbridge vapor sensor, IEEE Trans. Electron Devices, ED-33 (1986) 499-506.

[6] M.W. Putty, S.C. Chang, R.T. Howe, A.L. Robinson and K.D. Wise, One-port active polysilicon resonant microstructures, Proc. IEEE Micro Electro Mechanical Systems, Salt Lake City, UT, USA, Feb. 20-22, 1989, pp. 60-65.

[7] C. Linder, E. Zimmerman and N.F. de Rooij, Capacitive polysilicon resonator with MOS detection circuit, Sensors and Actuators A, 25-27 (1991) 591-595.

[8] M. Andrews, I. Harris and G. Turner, A comparison of squeezefilm theory with measurements on a microstructure, Sensors and Actuators A, 36 (1993) 79-87.

[9] K. Ikeda, H. Kuwayama, T. Kobayashi, T. Watanabe, T. Nishikawa and $T$. Yoshida, Three dimensional micromachining of silicon resonant strain gauge, Proc. 7th Sensor Symp., Tokyo, Japan, 1988, pp. 193-196.

[10] K. Ikeda, H. Kuwayama, T. Kobayashi, T. Watanabe, T. Nishikawa and T. Yoshida, Silicon pressure sensor with resonant strain gauge built into diaphragm, Proc. 7th Sensor Symp., Tokyo, Japan, 1988, Pp. 55-58.

[11] H. Guckel, J.J. Sniegowski, T.R. Christenson and F. Raissi, The application of fine-grained, tensile polysilicon to mechanically resonant transducers, Sensors and Actuators, $A 21-A 23$ (1990) 346-351.

[12] J.J. Sniegowski, H. Guckel and T.R. Christenson, Performance characteristics of second generation polysilicon resonating beam force transducers, Proc. IEEE Solid-State Sensors and Actuators Workshop, Hilkon Head Island, SC, USA, June 4-7, 1990, pp. 9-12.

[13] H.A.C. Tilmans and R. Legtenberg, Electrostatically driven vacuum-encapsulated polysilicon resonators; Part II - Theory and performance, Sensors and Actuators A, 44 (1994).

[14] H.A.C. Tilmans, D.J. IJntema and J.H.J. Fluitman, Single element excitation and detection of (micro-) mechanical resonators, Proc. 6th Int. Conf., Solid-State Sensors and Actuators (Trunsulucers '91), San Francisco, USA, June 24-28, 1991, pp. 533-537.

[15] H. Guckel, D.W. Burns, H.A.C. Tilmans, D.W. DeRoo and C.R. Rutigliano, Mechanical properties of fine grained polysilicon; the repeatability issue, IEEE Solid-State Sensors Workshop, Hilton Head Island, SC, USA, June 6-9, 1988, pp. 96-99.

[16] S.P. Timoshenko and S. Woinowsky-Krieger, Theory of Plates and Shells, McGraw-Hill, New York, 2nd edn., 1970, p. 202. 
[17] H. Guckel and D.W. Burns, A technology for integrated transducers, Proc. 3rd Int. Conf. Solid State Sensors and Actuators (Transducess '85), Philadelphia, PA, USA, June 11-14, 1985, pp. 90-92.

[18] R.T. Howe, Surface micromaching for microsensors and microactuators, J. Vac. Sci. Technol B, 6 (6) (1988) 1809-1813.

[19] D.W. Burns, Micromechanics of integrated sensors and the planar processed pressure transducers, Ph.D. Thesis, Dept. of Materials Science, University of Wisconsin, Madison, WI, USA, 1988.

[20] R.T. Howe and R.S. Muller, Stress in polycrystalline and amorphous silicon thin films, J. Appl. Phys., 54 (8) (1983) 4674-4675.

[21] T.I. Kamins, Design properties of polycrystalline silicon, Sensors and Actuators, A21-A23 (1990) 817-824.

[22] J.C. North, A.C. Adam and G.F. Richards, Ion implantation doping of polycrystalline silicon, Electrochem. Soc. Fall Meet, Pittsburgh, PA, USA, Extended Abstr. 78-2, 1978, p. 542.

[23] P. Scheeper, J.A. Voorthuyzen and P. Bergveld, Surface forces in micromachined structures, Tech. Digest, MME 90 , 2nd Workshop Micromachining, Micromechanics Microsystems, Berlin, Germany, Nov. 26-27, 1990, pp. 47-52.

[24] H. Guckel, J.J. Sniegowski, T.R. Christenson, S. Mohney and T.F. Kelly, Fabrication of micromechanical devices from polysilicon films with smooth surfaces, Sensors and Actuators, 20 (1989) 117-122.

[25] R. Legtenberg, H.A.C. Tilmans, J. Elders and M. Elwenspoek, Stiction of surface micromachined structures after rinsing and drying: model and investigation of adhesion mechanisms, Sensors and Actuators A, 43 (1994) 230-238.

[26] A. Boyde and C. Wood, Preparation of animal tissues for surface-scanning clectron microscopy, J. Microsc., 90 (1969) 221-249.

[27] N. Takeshima, K.J. Gabriel, M. Ozaki, J. Takahasji, H. Horiguchi and H. Fujita, Electrostatic parallelogram actuators, Proc. 6th Int. Conf. Solid-State Sensors and Actuators (Transducers '91), San Francisco, CA, USA, June 24-28, 1991, pp. 63-66.

[28] F.R. Blom, S. Bouwstra, M. Elwenspoek and J.H.J. Fluitman, Dependence of the quality factor of micromachined silicon beam resonators on pressure and geometry, J. Vac. Sci. Technol. B, 10 (1992) 19-26.

[29] K.F. Roenigk and K.F. Jensen, Low pressure CVD of silicon nitride, J. Electrochem. Soc., 134 (1987) 1777-1885.

[30] J.D. Zook, D.W. Burns, H. Guckel, J.J. Sniegowski, R.L. Engelstad and Z. Feng, Characteristics of polysilicon resonant microbeams, Sensors and Actuators $A, 35$ (1992) 51-59.

\section{Biographies}

Rob Legtenberg was born on June 21, 1964, in Hengelo, The Netherlands. He received the B.Sc. degree in applied physics from the Hogere Technische School Enschede, Enschede, The Netherlands, in June 1985. After fulfilling bis military service, he joined the MicroMechanical Research Group at the University of Twente as a process engineer in September 1986. Since April 1992 he has been preparing for his Ph.D., dealing with the development of actuators based on surfacemicromachining technology.

Harrie Tilmans was born on November 14, 1957, in Elsloo, The Netherlands. He received his M.Sc. degree in electrical engineering from the University of Twente, Enschede, The Netherlands, in May 1984. In June 1984 he became a temporary researcher at the University of Twente, where he worked on the development of a resonating force sensor. In April 1985 he joined the Faculty of Electrical and Computer Engineering of Boston University, Boston, MA, USA, as a visiting instructor. In August 1986 he became a research assistant at the Wisconsin Center for Applied Microelectronics at the University of Wisconsin, Madison, WI, USA, where he worked on the development of a CMOS process and on the development of polysilicon micromechanical resonators. In August 1988 be returned to The Netherlands, where he joined the MESA Research Institute of the University of Twente as a research associate to work on 'micromechanical sensors using encapsulated built-in resonant strain gauges'. He received his Ph.D. degree on this topic in January 1993. From August 1989 till January 1990 he wás on leave from the university at the Sensors Group of the Controls Research Department of Johnson Controls Inc., Milwaukee, WI, USA, working on a low-range differential pressure sensor. From September 1, 1992 till August 1, 1993 he was employed by FOM (Foundation for Fundamental Research on Matter, The Netherlands) in a postdoctorate position within the Micromechanics Group of the MESA Research Institute to work on the characterization of mechanical properties of thin films. He is currently employed by the Catholic University of Leuven in Belgium to continue his work on microsystems technology. 